\title{
Guaranteed Cost Control for Exponential Synchronization of Cellular Neural Networks with Mixed Time-Varying Delays via Hybrid Feedback Control
}

\author{
T. Botmart ${ }^{1,2}$ and W. Weera ${ }^{3}$ \\ ${ }^{1}$ Department of Mathematics, Srinakharinwirot University, Bangkok 10110, Thailand \\ ${ }^{2}$ Centre of Excellence in Mathematics, CHE, Si Ayutthaya Road, Bangkok 10400, Thailand \\ ${ }^{3}$ Department of Mathematics, Chiang Mai University, Chiang Mai 50200, Thailand
}

Correspondence should be addressed to T. Botmart; thongchaib@swu.ac.th

Received 30 November 2012; Revised 9 February 2013; Accepted 18 February 2013

Academic Editor: Yanni Xiao

Copyright (C) 2013 T. Botmart and W. Weera. This is an open access article distributed under the Creative Commons Attribution License, which permits unrestricted use, distribution, and reproduction in any medium, provided the original work is properly cited.

The problem of guaranteed cost control for exponential synchronization of cellular neural networks with interval nondifferentiable and distributed time-varying delays via hybrid feedback control is considered. The interval time-varying delay function is not necessary to be differentiable. Based on the construction of improved Lyapunov-Krasovskii functionals is combined with LeibnizNewton's formula and the technique of dealing with some integral terms. New delay-dependent sufficient conditions for the exponential synchronization of the error systems with memoryless hybrid feedback control are first established in terms of LMIs without introducing any free-weighting matrices. The optimal guaranteed cost control with linear error hybrid feedback is turned into the solvable problem of a set of LMIs. A numerical example is also given to illustrate the effectiveness of the proposed method.

\section{Introduction}

In the past decade, synchronization in neural networks (NNs), such as cellular NNs, Hopfield NNs, and bidirectional associative memory networks, has received a great deal of interest among scientists in a variety of areas, such as signal processing, pattern recognition, static image processing, associative memory, content-addressable memory and combinatorial optimization [1-6]. In performing a periodicity or stability analysis of a neural network, the conditions to be imposed on the neural network are determined by the characteristics of various activation functions and network parameters. When neural networks are created for problem solving, it is desirable for their activation functions not to be too restrictive. As a result, there has been considerable research work on the stability of neural networks with various activation functions and more general conditions [7-9]. On the other hand, the problem of chaos synchronization has attracted a wide range of research activity in recent years. A chaotic system has complex dynamical behaviors that possess some special features, such as being extremely sensitive to tiny variations of initial conditions and having bounded trajectories in the phase space. The first concept of chaos synchronization making two chaotic systems oscillate in a synchronized manner was introduced by [2], and many different methods have been applied theoretically and experimentally to synchronize chaotic systems, for example, linear feedback method [10], active control [11], adaptive control [11, 12], impulsive control $[13,14]$, back-stepping design [15], timedelay feedback control [16] and intermittent control [17], sampled data control [18], and so forth.

The guaranteed cost control of uncertain systems was first put forward by Chang and Peng [19] and introduced by a lot of authors, which is to design a controller to robustly stabilize the uncertain system and guarantee an adequate level of performance. The guaranteed cost control approach has recently been extended to the neural networks with time delay (see [7, 9, 20-22] and references cited therein). In [7], author investigated the guaranteed cost control problem for a class of neural networks with various activation functions 
and mixed time-varying delays in state and control. By using improved Lyapunov-Krasovskii functionals combined with LMIs technique. A delay-dependent criterion for existence of the guaranteed cost controller is derived in terms of LMIs. Optimal guaranteed cost control for linear systems with mixed interval nondifferentiable time-varying delayed state and control has been studied in [20]. By constructing a set of augmented Lyapunov-Krasovskii functionals combined with Newton-Leibniz formula, the sufficient conditions for the existence of an optimal guaranteed cost state feedback for the system have been derived in terms of LMIs. Moreover, all this work has been developed for the guaranteed cost control synchronization of time-varying delay systems [21-24]. Based on the Lyapunov-Krasovskii analysis process and the zoned discussion and maximax synthesis (ZDMS) method, the quadratic matrix inequality (QMI) criterion for the guaranteed cost synchronous controller is designed to synchronize the given neural networks with time-varying delay [21, 22]. However, to the best of our knowledge, few published papers deal with the problem of guaranteed cost synchronization of cellular neural networks with time-varying delay by using feedback control. So, our paper presents cellular neural networks with various activation functions and mixed timevarying delays and we also approach to establish both delay and nondelay controllers to the system.

It is known that exponential stability is a more favorite property than asymptotic stability since it gives a faster convergence rate to the equilibrium point and any information about the decay rates of the delayed neural networks. Therefore it is particularly important when the exponential convergence rate is used to determine the speed of neural computations. The exponential stability property guarantees that, whatever transformation occurs, the network's ability to store rapidly the activity pattern is left invariant by self-organization. Thus, it is important to determine the exponential stability and to estimate the exponential convergence rate for delayed neural networks. Recently, exponential synchronization of neural networks has been widely investigated and many effective methods have been presented by [25-33] A synchronization scheme for a class of delayed neural networks with time-varying delays based on the Lyapunov functional method and Hermitian matrices theory is derived in [25]. In [26, 27], authors presented sufficient conditions for the exponential synchronization of neural networks with time-varying delays in terms of the feasible solution to the LMIs.

The stability criteria for system with time delays can be classified into two categories: delay independent and delay dependent. Delay-independent criteria do not employ any information on the size of the delay, while delay-dependent criteria make use of such information at different levels. Delay-dependent stability conditions are generally less conservative than delay-independent ones especially when the delay is small. Recently, delay-dependent stability for interval time-varying delay was investigated in [8, 9, 34-37]. Interval time-varying delay is a time delay that varies in an interval in which the lower bound is not restricted to be 0 . Tian and Zhou [36] considered the delay-dependent asymptotic stability criteria for neural networks (NNs) with time-varying interval delay. By introducing a novel Lyapunov functional stability criteria of asymptotic stability is derived in terms of LMIs with adding the term free-weighting matrix. Delaydependent robust exponential stabilization criteria for interval time-varying delay systems are proposed in [37], by using Lyapunov-Krasovskii functionals combined with the freeweighting matrices. It is noted that the former has more matrix variables than our result. Therefore, our result has less conservative and matrix variables than [36, 37]. Moreover, neural networks with distributed delays have been extensively discussed [29-33, 38-41]. In [38, 39, 41], a neural circuit has been designed with distributed delays, which solves the general problem of recognized patterns in a time-dependent signal. The master-slave synchronization problem has been investigated for neural networks with discrete and distributed time-varying delays in $[29,30]$; based on the drive-response concept, LMI approach, and the Lyapunov stability theorem, several delay-dependent feedback controllers were derived to achieve the exponential synchronization of the chaotic neural networks. In [33], by constructing proper LyapunovKrasovskii functional and employing a combination of the free-weighting matrix method, Leibniz-Newton, formulation and inequality technique, the feedback controllers were derived to ensure the asymptotical and exponential synchronization of the addressed neural networks.

However, It is worth pointing out that the given criteria in $[21,22]$ still have been based on the following conditions: (1) the time-varying delays are continuously differentiable; (2) the derivative of time-varying delay is bounded; (3) the time-varying delays with the lower bound are restricted to be 0 . However, in most cases, these conditions are difficult to satisfy. Therefore, in this paper we will employ some new techniques so that the above conditions can be removed. To the best of our knowledge, the guaranteed cost synchronization problem of the cellular neural networks with nondifferentiable interval time-varying discrete and distributed delays and various activation functions is seldom discussed in terms of LMIs, which remains important and challenging.

In this paper, inspired by the above discussions, we consider the problem of guaranteed cost control for exponential synchronization of cellular neural networks with interval nondifferentiable and distributed time-varying delays via hybrid feedback control. There are various activation functions which are considered in the system, and the restriction on differentiability of interval time-varying delays is removed, which means that a fast interval time-varying delay is allowed. Based on the construction of improved LyapunovKrasovskii functionals combined with Liebniz-Newton formula and the technique of dealing with some integral terms, new delay-dependent sufficient conditions for the exponential stabilization of the memoryless feedback controls are first established of LMIs without introducing any freeweighting matrices. The optimal guaranteed cost control with linear error hybrid feedback is turned into the solvable problem of a set of LMIs. The new stability condition is much less conservative and more general than some existing results. A numerical example is also given to illustrate the effectiveness of the proposed method. 
The rest of this paper is organized as follows. In Section 2, we give notations, definition, propositions, and lemma for using in the proof of the main results. Delay-dependent sufficient conditions of guaranteed cost control for exponential synchronization of cellular neural networks with various activation functions and interval and distributed timevarying delays with memoryless hybrid feedback controls are presented in Section 3. Numerical examples illustrating the obtained results are given in Section 4. The paper ends with conclusions in Section 5 and cited references.

\section{Preliminaries}

The following notation will be used in this paper: $\mathbb{R}^{+}$denotes the set of all real nonnegative numbers; $\mathbb{R}^{n}$ denotes the $n$ dimensional space and the vector norm $\|\cdot\| ; M^{n \times r}$ denotes the space of all matrices of $(n \times r)$-dimensions.

$A^{T}$ denotes the transpose of matrix $A ; A$ is symmetric if $A=A^{T} ; I$ denotes the identity matrix; $\lambda(A)$ denotes the set of all eigenvalues of $A ; \lambda_{\max }(A)=\max \{\operatorname{Re} \lambda ; \lambda \in \lambda(A)\}$.

$x_{t}:=\{x(t+s): s \in[-h, 0]\},\left\|x_{t}\right\|=\sup _{s \in[-h, 0]} \| x(t+$ $s) \| ; C\left([0, t], \mathbb{R}^{n}\right)$ denotes the set of all $\mathbb{R}^{n}$-valued continuous functions on $[0, t] ; L_{2}\left([0, t], \mathbb{R}^{m}\right)$ denotes the set of all the $\mathbb{R}^{m}$ valued square integrable functions on $[0, t]$.

Matrix $A$ is called semipositive definite $(A \geq 0)$ if $\langle A x, x\rangle \geq 0$ for all $x \in \mathbb{R}^{n} ; A$ is positive definite $(A>0)$ if $\langle A x, x\rangle>0$ for all $x \neq 0 ; A>B$ means $A-B>0$. The symmetric term in a matrix is denoted by $*$.

In this paper, the master-slave cellular neural networks (MSCNNs) with mixed time-varying delays are described as follows:

$$
\begin{gathered}
\dot{x}(t)=-A x(t)+C \tilde{f}(x(t))+D \tilde{g}\left(x\left(t-h_{1}(t)\right)\right) \\
+E \int_{t-k_{1}(t)}^{t} \widetilde{h}(x(s)) d s+I(t), \\
x(t)=\phi_{1}(t), \quad t \in[-d, 0], \\
\dot{y}(t)=-A y(t)+C \tilde{f}(y(t))+D \tilde{g}\left(y\left(t-h_{1}(t)\right)\right) \\
+E \int_{t-k_{1}(t)}^{t} \tilde{h}(y(s)) d s+I(t)+\mathscr{U}(t), \\
y(t)=\phi_{2}(t), \quad t \in[d, 0],
\end{gathered}
$$

where $x(t)=\left[x_{1}(t), x_{2}(t), \ldots, x_{n}(t)\right] \in \mathbb{R}^{n}$ and $y(t)=\left[y_{1}(t)\right.$, $\left.y_{2}(t), \ldots, y_{n}(t)\right] \in \mathbb{R}^{n}$ are the master system's state vector and the slave system's state vector of the neural networks, respectively. $n$ is the number of neural,

$$
\begin{aligned}
& \tilde{f}(x(t))=\left[\tilde{f}_{1}\left(x_{1}(t)\right), \tilde{f}_{2}\left(x_{2}(t)\right), \ldots, \widetilde{f}_{n}\left(x_{n}(t)\right)\right]^{T}, \\
& \tilde{g}(x(t))=\left[\widetilde{g}_{1}\left(x_{1}(t)\right), \widetilde{g}_{2}\left(x_{2}(t)\right), \ldots, \widetilde{g}_{n}\left(x_{n}(t)\right)\right]^{T}, \\
& \widetilde{h}(x(t))=\left[\widetilde{h}_{1}\left(x_{1}(t)\right), \widetilde{h}_{2}\left(x_{2}(t)\right), \ldots, \widetilde{h}_{n}\left(x_{n}(t)\right)\right]^{T},
\end{aligned}
$$

are the activation functions, $A=\operatorname{diag}\left(\bar{a}_{1}, \bar{a}_{2}, \ldots, \bar{a}_{n}\right), \bar{a}_{i}>0$ represents the self-feedback term, and $C, D$, and $E$ denote the connection weights, the discretely delayed connection weights, and the distributively delayed connection weight, respectively.

The synchronization error $e(t)$ is the form $e(t)=y(t)-$ $x(t)$. Therefore, the cellular neural networks with mixed timevarying delays of synchronization error between the masterslave systems given in (1) and (2) can be described by

$$
\begin{aligned}
& \dot{e}(t)=-A e(t)+C f(e(t))+D g\left(e\left(t-h_{1}(t)\right)\right) \\
&+E \int_{t-k_{1}(t)}^{t} h(e(s)) d s+\mathcal{U}(t), \\
& e(t)=\phi_{2}(t)-\phi_{1}(t)=\phi(t), \quad t \in[-d, 0],
\end{aligned}
$$

where $f(e(t))=\tilde{f}(e(t)+x(t))-\tilde{f}(x(t)), g\left(e\left(t-h_{1}(t)\right)\right)=$ $\tilde{g}\left(e\left(t-h_{1}(t)\right)+x\left(t-h_{1}(t)\right)\right)-\tilde{g}\left(x\left(t-h_{1}(t)\right)\right)$, and $\int_{t-k_{1}(t)}^{t} h(e(s)) d s=\int_{t-k_{1}(t)}^{t} h(e(s)+x(s))-h(x(s)) d s$. The state hybrid feedback controller $\mathcal{U}(t)$ satisfies $(\mathrm{H} 1)$ :

$$
\begin{aligned}
(\mathrm{H} 1): \mathcal{U}(t)= & B_{1} u(t)+B_{2} u\left(t-h_{2}(t)\right) \\
& +B_{3} \int_{t-k_{2}(t)}^{t} u(s) d s, \quad \forall t \geq 0,
\end{aligned}
$$

where $u(t)=K e(t)$ and $K$ is a constant matrix control gain. In this paper, our goal is to design suitable $K$ such that system (2) synchronizes with system (1). Then, substituting it into (4), it is easy to get the following:

$$
\begin{aligned}
\dot{e}(t)= & -A e(t)+C f(e(t))+D g\left(e\left(t-h_{1}(t)\right)\right) \\
& +E \int_{t-k_{1}(t)}^{t} h(e(s)) d s+B_{1} K e(t)+B_{2} K e\left(t-h_{2}(t)\right) \\
& +B_{3} K \int_{t-k_{2}(t)}^{t} e(s) d s, \quad \forall t \geq 0, \\
& e(t)=\phi_{2}(t)-\phi_{1}(t)=\phi(t), \quad t \in[-d, 0] .
\end{aligned}
$$

Throughout this paper, we consider various activation functions and the activation functions $\widetilde{f}(\cdot), \widetilde{g}(\cdot)$, and $\widetilde{h}(\cdot)$ satisfy the following assumption.

(A1) The activation functions $\widetilde{f}(\cdot), \widetilde{g}(\cdot)$, and $\widetilde{h}(\cdot)$ satisfy Lipschitzian with the Lipschitz constants $\widehat{f}_{i}, \widehat{g}_{i}>0$, and $\widehat{h}_{i}>$ 0 :

$$
\begin{array}{rr}
\left|\widetilde{f}_{i}\left(\xi_{1}\right)-\widetilde{f}_{i}\left(\xi_{2}\right)\right| \leq \widehat{f}_{i}\left|\xi_{1}-\xi_{2}\right|, \quad & i=1,2, \ldots, n, \\
& \forall \xi_{1}, \xi_{2} \in \mathbb{R}, \\
\left|\widetilde{g}_{i}\left(\xi_{1}\right)-\widetilde{g}_{i}\left(\xi_{2}\right)\right| \leq \widehat{g}_{i}\left|\xi_{1}-\xi_{2}\right|, \quad & i=1,2, \ldots, n, \\
& \forall \xi_{1}, \xi_{2} \in \mathbb{R}, \\
\left|\widetilde{h}_{i}\left(\xi_{1}\right)-\widetilde{h}_{i}\left(\xi_{2}\right)\right| \leq \widehat{h}_{i}\left|\xi_{1}-\xi_{2}\right|, \quad & i=1,2, \ldots, n, \\
& \forall \xi_{1}, \xi_{2} \in \mathbb{R},
\end{array}
$$


and we denote that

$$
\begin{aligned}
& F=\operatorname{diag}\left\{\widehat{f}_{i}, i=1,2, \ldots, n\right\}, \\
& G=\operatorname{diag}\left\{\widehat{g}_{i}, i=1,2, \ldots, n\right\}, \\
& H=\operatorname{diag}\left\{\widehat{h}_{i}, i=1,2, \ldots, n\right\} .
\end{aligned}
$$

The time-varying delay functions $h_{i}(t)$ and $k_{i}(t), i=1,2$, satisfy the condition

$$
\begin{gathered}
0 \leq h_{1 m} \leq h_{1}(t) \leq h_{1 M}, \quad 0 \leq h_{2}(t) \leq h_{2}, \\
0 \leq k_{1}(t) \leq k_{1}, \quad 0 \leq k_{2}(t) \leq k_{2} .
\end{gathered}
$$

It is worth noting that the time delay is assumed to be a continuous function belonging to a given interval, which means that the lower and upper bounds for the time-varying delay are available, but the delay function is bounded but not restricted to being zero. The initial functions $\phi(t) \epsilon$ $C^{1}\left([-d, 0], \mathbb{R}^{n}\right), d=\max \left\{h_{1 M}, h_{2}, k_{1}, k_{2}\right\}$, with the norm

$$
\|\phi\|=\sup _{t \in[-d, 0]} \sqrt{\|\phi(t)\|^{2}+\|\dot{\phi}(t)\|^{2}} .
$$

Define the following quadratic cost function of the associated system (4) as follows:

$$
J=\int_{0}^{\infty}\left[e^{T}(t) Q_{1} e(t)+u^{T}(t) Q_{2} u(t)\right] d t,
$$

where $Q_{1} \in \mathbb{R}^{n \times n}$ and $Q_{2} \in \mathbb{R}^{m \times m}$ are positive definite matrices.

Remark 1. If $E=0, B_{2}=0, B_{3}=0$, and $f(\cdot)=g(\cdot)$, the system model (6) turns into the cellular neural networks with activation functions and time-varying delays proposed by [21, 22]

$$
\begin{array}{r}
\dot{e}(t)=-A e(t)+C f(e(t))+D f\left(e\left(t-h_{1}(t)\right)\right)+B_{1} K e(t), \\
\forall t \geq 0 .
\end{array}
$$

Therefore, (6) is a general cellular neural networks model, with (12) as the special case.

Definition 2. Given $\alpha>0$, the zero solution of system (6) with $u(t)=K e(t)$ is $\alpha$-stable if there exists a positive number $N>0$ such that every solution $e(t, \phi)$ satisfies the following condition:

$$
\|e(t, \phi)\| \leq N e^{-\alpha t}\|\phi\|, \quad \forall t \geq 0 .
$$

We introduce the following technical well-known propositions and lemma, which will be used in the proof of our results.

Proposition 3 (see [42] (Cauchy inequality)). For any symmetric positive definite matrix $N \in M^{n \times n}$ and $x, y \in \mathbb{R}^{n}$, we have

$$
\pm 2 x^{T} y \leq x^{T} N x+y^{T} N^{-1} y
$$

Proposition 4 (see [42]). For any symmetric positive definite matrix $M>0$, scalar $\gamma>0$, and vector function $\omega:[0, \gamma] \rightarrow$ $\mathbb{R}^{n}$ such that the integrations concerned are well defined, the following inequality holds:

$$
\left(\int_{0}^{\gamma} \omega(s) d s\right)^{T} M\left(\int_{0}^{\gamma} \omega(s) d s\right) \leq \gamma\left(\int_{0}^{\gamma} \omega^{T}(s) M \omega(s) d s\right) .
$$

Proposition 5 (see [42] (Schur complement lemma)). Given constant symmetric matrices $X, Y$, and $Z$ with appropriate dimensions satisfying $X=X^{T}, Y=Y^{T}>0$, then $X+$ $Z^{T} Y^{-1} Z<0$ if and only if

$$
\left(\begin{array}{cc}
X & Z^{T} \\
Z & -Y
\end{array}\right)<0 \quad \text { or }\left(\begin{array}{cc}
-Y & Z \\
Z^{T} & X
\end{array}\right)<0
$$

\section{Main Results}

Let us set

$$
\begin{aligned}
\lambda_{1}= & \lambda_{\min }\left(P^{-1}\right), \\
\lambda_{2}= & \lambda_{\max }\left(P^{-1}\right)+\left(h_{1 m}+h_{1 M}\right) \lambda_{\max }\left(P^{-1} Q P^{-1}\right) \\
& +\left(h_{1 m}^{3}+h_{1 M}^{3}\right) \lambda_{\max }\left(P^{-1} R P^{-1}\right) \\
& +\delta^{3} \lambda_{\max }\left(P^{-1} U P^{-1}\right)+h_{2}^{3} \lambda_{\max }\left(P^{-1} Y^{T} S_{1}^{-1} Y P^{-1}\right) \\
& +k_{1}^{2} \lambda_{\max }\left(H U_{3}^{-1} H\right)+k_{2}^{2} \lambda_{\max }\left(P^{-1} Y^{T} S_{2}^{-1} Y P^{-1}\right) .
\end{aligned}
$$

Theorem 6. Given $\alpha>0, Q_{1}>0$ and $Q_{2}>0, u(t)=K e(t)$ is a guaranteed cost controller if there exist symmetric positive definite matrices $P, Q, R, U, S_{1}$, and $S_{2}$, diagonal matrices $U_{i}, i=1,2,3$, and a matrix $Y$ with appropriately dimensioned such that the following LMIs holds:

$$
\begin{aligned}
& \Gamma_{1}=\Gamma-\left[\begin{array}{lllll}
0 & 0 & -I & I & 0
\end{array}\right]^{T} \times e^{-2 \alpha h_{1 M}} U\left[\begin{array}{lllll}
0 & 0 & -I & I & 0
\end{array}\right]<0, \\
& \Gamma_{2}=\Gamma-\left[\begin{array}{lllll}
0 & 0 & 0 & I & -I
\end{array}\right]^{T} \times e^{-2 \alpha h_{1 M}} U\left[\begin{array}{lllll}
0 & 0 & 0 & I & -I
\end{array}\right]<0, \\
& \Gamma_{3}= \\
& {\left[\begin{array}{cccccc}
-0.1\left(e^{-2 \alpha h_{1 m}}+e^{-2 \alpha h_{1 M}}\right) R & 2 P F^{T} & P H^{T} & 2 Y^{T} & P Q_{1} & Y^{T} Q_{2} \\
* & -2 U_{1} & 0 & 0 & 0 & 0 \\
* & * & -U_{3} & 0 & 0 & 0 \\
* & * & * & -2 e^{-2 \alpha h_{2}} S_{1} & 0 & 0 \\
* & * & * & * & -Q_{1} & 0 \\
* & * & * & * & * & -Q_{2}
\end{array}\right]} \\
& <0 \text {, } \\
& \Gamma_{4}=\left[\begin{array}{cc}
-0.1 P & h_{2}^{2} Y^{T} \\
* & -h_{2}^{2} S_{1}
\end{array}\right]<0,
\end{aligned}
$$




$$
\begin{gathered}
\Gamma_{5}=\left[\begin{array}{cccc}
-0.1 e^{-2 \alpha h_{1 M}} U & 2 P G^{T} \\
* & & -2 U_{2}
\end{array}\right]<0, \\
\Gamma=\left[\begin{array}{ccccc}
\Gamma_{11} & \Gamma_{12} & \Gamma_{13} & 0 & \Gamma_{15} \\
* & \Gamma_{22} & 0 & 0 & 0 \\
* & * & \Gamma_{33} & \Gamma_{34} & 0 \\
* & * & * & \Gamma_{44} & \Gamma_{45} \\
* & * & * & * & \Gamma_{55}
\end{array}\right],
\end{gathered}
$$

where

$$
\begin{aligned}
& \Gamma_{11}=[-A+\alpha I] P+P[-A+\alpha I]^{T}-B Y-Y^{T} B^{T} \\
& +2 Q+C^{T} U_{1} C+D^{T} U_{2} D+k_{1} e^{2 \alpha k_{1}} E^{T} U_{3} E \\
& +3 e^{2 \alpha h_{2}} B_{2}^{T} S_{1} B_{2}+2 k_{2} e^{2 \alpha k_{2}} B_{3}^{T} S_{2} B_{3} \\
& -0.9 e^{-2 \alpha h_{1 m}} R-0.9 e^{-2 \alpha h_{1 M}} R, \\
& \Gamma_{12}=-P A^{T}-Y^{T} B^{T} \\
& \Gamma_{13}=e^{-2 \alpha h_{1 m}} R \text {, } \\
& \Gamma_{15}=e^{-2 \alpha h_{1 M}} R \text {, } \\
& \Gamma_{22}=h_{1 m}^{2} R+h_{1 M}^{2} R+\delta^{2} U-1.9 P \\
& +C^{T} U_{1} C+D^{T} U_{2} D+2 k_{1} e^{2 \alpha k_{1}} E^{T} U_{3} E \\
& +3 e^{2 \alpha h_{2}} B_{2}^{T} S_{1} B_{2}+2 k_{2} e^{2 \alpha k_{2}} B_{3}^{T} S_{2} B_{3} \\
& \Gamma_{33}=-e^{-2 \alpha h_{1 m}} Q-e^{-2 \alpha h_{1 m}} R-e^{-2 \alpha h_{1 M}} U \text {, } \\
& \Gamma_{34}=e^{-2 \alpha h_{1 M}} U \text {, } \\
& \Gamma_{44}=-1.9 e^{-2 \alpha h_{1 M}} U, \\
& \Gamma_{45}=e^{-2 \alpha h_{1 M}} U \text {, } \\
& \Gamma_{55}=-e^{-2 \alpha h_{1 M}} Q-e^{-2 \alpha h_{1 M}} R-e^{-2 \alpha h_{1 M}} U,
\end{aligned}
$$

then the error system (6) is exponentially stabilizable. Moreover, the feedback control is

$$
u(t)=-Y P^{-1} e(t), \quad t \geq 0,
$$

and the upper bound of the cost function (11) is as follows:

$$
J \leq J^{*}=\lambda_{2}\|\phi\|^{2}
$$

Proof. Let $W=P^{-1}$ and let $z(t)=W e(t)$. Using the feedback control (24), we consider the following Lyapunov-Krasovskii functional:

$$
V(t, e(t))=\sum_{i=1}^{9} V_{i},
$$

where

$$
\begin{aligned}
& V_{1}=e^{T}(t) W e(t), \\
& V_{2}=\int_{t-h_{1 m}}^{t} e^{2 \alpha(s-t)} e^{T}(s) W Q W e(s) d s, \\
& V_{3}=\int_{t-h_{1 M}}^{t} e^{2 \alpha(s-t)} e^{T}(s) W Q W e(s) d s, \\
& V_{4}=h_{1 m} \int_{-h_{1 m}}^{0} \int_{t+s}^{t} e^{2 \alpha(\theta-t)} \dot{e}^{T}(\theta) W R W \dot{e}(\theta) d \theta d s, \\
& V_{5}=h_{1 M} \int_{-h_{1 M}}^{0} \int_{t+s}^{t} e^{2 \alpha(\theta-t)} \dot{e}^{T}(\theta) W R W \dot{e}(\theta) d \theta d s, \\
& V_{6}=\delta \int_{-h_{1 M}}^{-h_{1 m}} \int_{t+s}^{t} e^{2 \alpha(\theta-t)} \dot{e}^{T}(\theta) W U W \dot{e}(\theta) d \theta d s, \\
& V_{7}=h_{2} \int_{h_{2}}^{0} \int_{t+s}^{t} e^{2 \alpha(\theta-t)} \dot{u}^{T}(\theta) S_{1}^{-1} \dot{u}(\theta) d \theta d s, \\
& V_{8}=\int_{-k_{1}}^{0} \int_{t+s}^{t} e^{2 \alpha(\theta-t)} h^{T}(e(\theta)) U_{3}^{-1} h(e(\theta)) d \theta d s, \\
& V_{9}=\int_{-k_{2}}^{0} \int_{t+s}^{t} e^{2 \alpha(\theta-t)} u^{T}(\theta) S_{2}^{-1} u(\theta) d \theta d s .
\end{aligned}
$$

It is easy to check that

$$
\lambda_{1}\|e(t)\|^{2} \leq V(t, e(t)) \leq \lambda_{2}\left\|e_{t}(t)\right\|^{2}, \quad \forall t \geq 0 .
$$

Taking the derivative of $V(t, e(t))$ along the solution of system (6), we have

$$
\begin{aligned}
& \dot{V}_{1}=2 e^{T}(t) W \dot{e}(t) \\
& =2 z^{T}(t)\left[-A e(t)+C f(e(t))+D g\left(e\left(t-h_{1}(t)\right)\right)\right. \\
& +E \int_{t-k_{1}(t)}^{t} h(e(s)) d s-B_{1} Y P^{-1} e(t) \\
& \left.+B_{2}(t) K e\left(t-h_{2}(t)\right)+B_{3} K \int_{t-k_{2}(t)}^{t} e(s) d s\right] \\
& =z^{T}(t)\left[-A P-P A^{T}-2 B_{1} Y\right] z(t)+2 z^{T}(t) C f(e(t)) \\
& +2 z^{T}(t) D g\left(e\left(t-h_{1}(t)\right)\right) \\
& +2 z^{T}(t) E \int_{t-k_{1}(t)}^{t} h(e(s)) d s \\
& +2 z^{T}(t) B_{2} u\left(t-h_{2}(t)\right) \\
& +2 z^{T}(t) B_{3} \int_{t-k_{2}(t)}^{t} u(s) d s,
\end{aligned}
$$




$$
\begin{gathered}
\dot{V}_{2}=z^{T}(t) Q z(t)-e^{-2 \alpha h_{1 m}} z^{T}\left(t-h_{1 m}\right) Q z\left(t-h_{1 m}\right) \\
\quad-2 \alpha V_{2}, \\
\begin{aligned}
\dot{V}_{3}= & z^{T}(t) Q z(t)-e^{-2 \alpha h_{1 M}} z^{T}\left(t-h_{1 M}\right) Q z\left(t-h_{1 M}\right) \\
& -2 \alpha V_{3},
\end{aligned} \\
\dot{V}_{4} \leq h_{1 m}^{2} \dot{z}^{T}(t) R \dot{z}(t)-h_{1 m} e^{-2 \alpha h_{1 m}} \int_{t-h_{1 m}}^{t} \dot{z}^{T}(s) R \dot{z}(s) d s \\
\quad-2 \alpha V_{4}, \\
\dot{V}_{5} \leq h_{1 M}^{2} \dot{z}^{T}(t) R \dot{z}(t)-h_{1 M} e^{-2 \alpha h_{1 M}} \int_{t-h_{1 M}}^{t} \dot{z}^{T}(s) R \dot{z}(s) d s \\
\quad-2 \alpha V_{5}, \\
\dot{V}_{6} \leq \delta^{2} \dot{z}^{T}(t) U \dot{z}(t)-\delta e^{-2 \alpha h_{1 M}} \int_{t-h_{1 M}}^{t-h_{1 m}} \dot{z}^{T}(s) R \dot{z}(s) d s \\
\quad-2 \alpha V_{6},
\end{gathered}
$$$$
\dot{V}_{7} \leq h_{2}^{2} \dot{u}^{T}(t) S_{1}^{-1} \dot{u}(t)-h_{2} e^{-2 \alpha h_{2}} \int_{t-h_{2}}^{t} \dot{u}^{T}(s) S_{1}^{-1} \dot{u}(s) d s
$$$$
-2 \alpha V_{7},
$$$$
\dot{V}_{8} \leq k_{1} h^{T}(e(t)) U_{3}^{-1} h(e(t))
$$$$
-e^{-2 \alpha k_{1}} \int_{t-k_{1}}^{t} h^{T}(e(s)) U_{3}^{-1} h(e(s)) d s-2 \alpha V_{8},
$$$$
\dot{V}_{9} \leq k_{2}^{2} u^{T}(t) S_{2}^{-1} u(t)-k_{2} e^{-2 \alpha k_{2}} \int_{t-k_{2}}^{t} u^{T}(s) S_{2}^{-1} u(s) d s
$$$$
-2 \alpha V_{9} .
$$

For assumption (A1), we can obtain the following three inequalities:

$$
\begin{aligned}
\left|f_{i}\left(e_{i}(t)\right)\right| & =\left|\tilde{f}_{i}\left(e_{i}(t)+x_{i}(t)\right)-\tilde{f}_{i}\left(x_{i}(t)\right)\right| \\
& \leq \widehat{f}_{i}\left|e_{i}(t)+x_{i}(t)-x_{i}(t)\right|=\widehat{f}_{i}\left|e_{i}(t)\right|, \\
\left|g_{i}\left(e_{i}(t)\right)\right| & =\left|\widetilde{g}_{i}\left(e_{i}(t)+x_{i}(t)\right)-\widetilde{g}_{i}\left(x_{i}(t)\right)\right| \\
& \leq \widehat{g}_{i}\left|e_{i}(t)+x_{i}(t)-x_{i}(t)\right|=\widehat{g}_{i}\left|e_{i}(t)\right|, \\
\left|h_{i}\left(e_{i}(t)\right)\right| & =\left|\widetilde{h}_{i}\left(e_{i}(t)+x_{i}(t)\right)-\widetilde{h}_{i}\left(x_{i}(t)\right)\right| \\
& \leq \widehat{h}_{i}\left|e_{i}(t)+x_{i}(t)-x_{i}(t)\right|=\widehat{h}_{i}\left|e_{i}(t)\right| .
\end{aligned}
$$

Applying Propositions 3 and 4 and since the matrices $U_{i}, i=$ $1,2,3$ are diagonal, we have

$$
\begin{aligned}
& 2 z^{T}(t) C f(e(t)) \\
& \quad \leq z^{T}(t) C^{T} U_{1} C z(t)+f^{T}(e(t)) U_{1}^{-1} f(e(t))
\end{aligned}
$$

$$
\begin{aligned}
& \leq z^{T}(t) C^{T} U_{1} C z(t)+e^{T}(t) F^{T} U_{1}^{-1} F e(t) \\
& =z^{T}(t) C^{T} U_{1} C z(t)+z^{T}(t) P F^{T} U_{1}^{-1} F P z(t), \\
& 2 z^{T}(t) D g\left(e\left(t-h_{1}(t)\right)\right) \\
& \leq z^{T}(t) D^{T} U_{2} D z(t)+g^{T}\left(e\left(t-h_{1}(t)\right)\right) U_{2}^{-1} \\
& \times g\left(e\left(t-h_{1}(t)\right)\right) \\
& \leq z^{T}(t) D^{T} U_{2} D z(t)+e^{T}\left(t-h_{1}(t)\right) G^{T} U_{2}^{-1} \\
& \times G e\left(t-h_{1}(t)\right) \\
& =z^{T}(t) D^{T} U_{2} D z(t)+z^{T}\left(t-h_{1}(t)\right) P G^{T} U_{2}^{-1} \\
& \times G P z\left(t-h_{1}(t)\right), \\
& k_{1} h^{T}(e(t)) U_{3}^{-1} h(e(t)) \\
& \leq k_{1} e^{T}(t) H^{T} U_{3}^{-1} H e(t) \\
& =k_{1} z^{T}(t) P H^{T} U_{3}^{-1} H P z(t) \text {, } \\
& 2 z^{T}(t) E \int_{t-k_{1}(t)}^{t} h(e(s)) d s \\
& \leq 2 k_{1} e^{2 \alpha k_{1}} z^{T}(t) E^{T} U_{3} E z(t) \\
& +\frac{1}{2 k_{1}} e^{-2 \alpha k_{1}}\left(\int_{t-k_{1}(t)}^{t} h(e(s)) d s\right)^{T} \\
& \times U_{3}^{-1}\left(\int_{t-k_{1}(t)}^{t} h(e(s)) d s\right) \\
& \leq 2 k_{1} e^{2 \alpha k_{1}} z^{T}(t) E^{T} U_{3} E z(t) \\
& +\frac{e^{-2 \alpha k_{1}}}{2} \int_{t-k_{1}(t)}^{t} h^{T}(e(s)) U_{3}^{-1} h(e(s)) d s, \\
& 2 z^{T}(t) B_{2} u\left(t-h_{2}(t)\right) \\
& \leq 3 e^{2 \alpha h_{2}} z^{T}(t) B_{2}^{T} S_{1} B_{2} z(t) \\
& +\frac{e^{-2 \alpha h_{2}}}{3} u^{T}\left(t-h_{2}(t)\right) S_{1}^{-1} u\left(t-h_{2}(t)\right),
\end{aligned}
$$$$
2 z^{T}(t) B_{3} \int_{t-k_{2}(t)}^{t} u(s) d s
$$$$
\leq 2 k_{2} e^{2 \alpha k_{2}} z^{T}(t) B_{3}^{T} S_{2} B_{3} z(t)
$$$$
+\frac{e^{-2 \alpha k_{2}}}{2 k_{2}}\left(\int_{t-k_{2}(t)}^{t} u(s) d s\right)^{T} S_{2}^{-1}\left(\int_{t-k_{1}(t)}^{t} u(s) d s\right)
$$$$
\leq 2 k_{2} e^{2 \alpha k_{2}} z^{T}(t) B_{3}^{T} S_{2} B_{3} z(t)
$$$$
+\frac{e^{-2 \alpha k_{2}}}{2} \int_{t-k_{2}(t)}^{t} u^{T}(s) S_{2}^{-1} u(s) d s,
$$

$h_{2}^{2} \dot{u}^{T}(t) S_{1}^{-1} \dot{u}(t)$ 


$$
\begin{aligned}
& =h_{2}^{2} \dot{e}^{T}(t) P^{-1} Y^{T} S_{1}^{-1} Y P^{-1} \dot{e}(t) \\
& =h_{2}^{2} \dot{z}^{T}(t) Y^{T} S_{1}^{-1} Y \dot{z}(t)
\end{aligned}
$$

and the Leibniz-Newton formula gives

$$
\begin{aligned}
- & h_{2} e^{-2 \alpha h_{2}} \int_{t-h_{2}}^{t} \dot{u}^{T}(s) S_{1}^{-1} \dot{u}(s) d s \\
\leq & -h_{2}(t) e^{-2 \alpha h_{2}} \int_{t-h_{2}(t)}^{t} \dot{u}^{T}(s) S_{1}^{-1} \dot{u}(s) d s \\
\leq & -e^{-2 \alpha h_{2}}\left(\int_{t-h_{2}(t)}^{t} \dot{u}(s) d s\right)^{T} S_{1}^{-1}\left(\int_{t-h_{2}(t)}^{t} \dot{u}(s) d s\right) \\
\leq & -e^{-2 \alpha h_{2}} u^{T}(t) S_{1}^{-1} u(t)+2 e^{-2 \alpha h_{2}} u^{T}(t) S_{1}^{-1} u\left(t-h_{2}(t)\right) \\
& -e^{-2 \alpha h_{2}} u^{T}\left(t-h_{2}(t)\right) S_{1}^{-1} u\left(t-h_{2}(t)\right) \\
\leq & -e^{-2 \alpha h_{2}} u^{T}(t) S_{1}^{-1} u(t)+3 e^{-2 \alpha h_{2}} u^{T}(t) S_{1}^{-1} u(t) \\
& +\frac{e^{-2 \alpha h_{2}}}{3} u^{T}\left(t-h_{2}(t)\right) S_{1}^{-1} S_{1} S_{1}^{-1} u\left(t-h_{2}(t)\right) \\
& -e^{-2 \alpha h_{2}} u^{T}\left(t-h_{2}(t)\right) S_{1}^{-1} u\left(t-h_{2}(t)\right) \\
= & 2 e^{-2 \alpha h_{2}} z^{T}(t) Y^{T} S_{1}^{-1} Y z(t) \\
& +\frac{e^{-2 \alpha h_{2}}}{3} u^{T}\left(t-h_{2}(t)\right) S_{1}^{-1} u\left(t-h_{2}(t)\right) \\
& -e^{-2 \alpha h_{2}} u^{T}\left(t-h_{2}(t)\right) S_{1}^{-1} u\left(t-h_{2}(t)\right) .
\end{aligned}
$$

Applying Proposition 4 and the Leibniz-Newton formula, we have

$$
\begin{aligned}
& -h_{1 m} e^{-2 \alpha h_{1 m}} \int_{t-h_{1 m}}^{t} \dot{z}^{T}(s) R \dot{z}(s) d s \\
& \leq-e^{-2 \alpha h_{1 m}}\left[\int_{t-h_{1 m}}^{t} \dot{z}(s)\right]^{T} R\left[\int_{t-h_{1 m}}^{t} \dot{z}(s)\right] \\
& \leq-e^{-2 \alpha h_{1 m}}\left[z(t)-z\left(t-h_{1 m}\right)\right]^{T} R\left[z(t)-z\left(t-h_{1 m}\right)\right] \\
& =-e^{-2 \alpha h_{1 m}}\left[z^{T}(t) R z(t)-2 z^{T}(t) R z\left(t-h_{1 m}\right)\right. \\
& \left.\quad+z^{T}\left(t-h_{1 m}\right) R z\left(t-h_{1 m}\right)\right], \\
& -h_{1 M} e^{-2 \alpha h_{1 M}} \int_{t-h_{1 M}}^{t} \dot{z}^{T}(s) R \dot{z}(s) d s \\
& \leq-e^{-2 \alpha h_{1 M}}\left[\int_{t-h_{1 M}}^{t} \dot{z}(s)\right]^{T} R\left[\int_{t-h_{1 M}}^{t} \dot{z}(s)\right] \\
& \leq-e^{-2 \alpha h_{1 M}}\left[z(t)-z\left(t-h_{1 M}\right)\right]^{T} R\left[z(t)-z\left(t-h_{1 M}\right)\right]
\end{aligned}
$$

$$
\begin{gathered}
=-e^{-2 \alpha h_{1 M}}\left[z^{T}(t) R z(t)-2 z^{T}(t) R z\left(t-h_{1 M}\right)\right. \\
\left.+z^{T}\left(t-h_{1 M}\right) R z\left(t-h_{1 M}\right)\right] .
\end{gathered}
$$

Note that

$$
\begin{aligned}
-\delta \int_{t-h_{1 M}}^{t-h_{1 m}} \dot{z}^{T}(s) U \dot{z}(s) d s \\
=-\left(h_{1 M}-h_{1 m}\right) \int_{t-h_{1 M}}^{t-h(t)} \dot{z}^{T}(s) U \dot{z}(s) d s \\
\quad-\left(h_{1 M}-h_{1 m}\right) \int_{t-h(t)}^{t-h_{1 m}} \dot{z}^{T}(s) U \dot{z}(s) d s \\
=-\left(h_{1 M}-h(t)\right) \int_{t-h_{1 M}}^{t-h(t)} \dot{z}^{T}(s) U \dot{z}(s) d s \\
\quad-\left(h(t)-h_{1 m}\right) \int_{t-h_{1 M}}^{t-h(t)} \dot{z}^{T}(s) U \dot{z}(s) d s \\
\quad-\left(h(t)-h_{1 m}\right) \int_{t-h(t)}^{t-h_{1 m}} \dot{z}^{T}(s) U \dot{z}(s) d s \\
\quad-\left(h_{1 M}-h(t)\right) \int_{t-h(t)}^{t-h_{1 m}} \dot{z}^{T}(s) U \dot{z}(s) d s .
\end{aligned}
$$

Using Proposition 4 gives

$$
\begin{aligned}
-\left(h_{1 M}\right. & -h(t)) \int_{t-h_{1 M}}^{t-h(t)} \dot{z}^{T}(s) U \dot{z}(s) d s \\
\leq & -\left[\int_{t-h_{1 M}}^{t-h(t)} \dot{z}(s) d s\right]^{T} U\left[\int_{t-h_{1 M}}^{t-h(t)} \dot{z}(s) d s\right] \\
\leq & -\left[z(t-h(t))-z\left(t-h_{1 M}\right)\right]^{T} U \\
\times & {\left[z(t-h(t))-z\left(t-h_{1 M}\right)\right], } \\
-(h(t) & \left.-h_{1 m}\right) \int_{t-h(t)}^{t-h_{1 M}} \dot{z}^{T}(s) U \dot{z}(s) d s \\
\leq & -\left[\int_{t-h(t)}^{t-h_{1 m}} \dot{z}(s) d s\right]^{T} U\left[\int_{t-h(t)}^{t-h_{1 m}} \dot{z}(s) d s\right] \\
\leq & -\left[z\left(t-h_{1 m}\right)-z(t-h(t))\right]^{T} U \\
\times & \times\left[z\left(t-h_{1 m}\right)-z(t-h(t))\right] .
\end{aligned}
$$

Let $\beta=\left(h_{1 M}-h(t)\right) /\left(h_{1 M}-h_{1 m}\right) \leq 1$. Then

$$
\begin{aligned}
& -\left(h_{1 M}-h(t)\right) \int_{t-h(t)}^{t-h_{1 m}} \dot{z}^{T}(s) U \dot{z}(s) d s \\
& =-\beta \int_{t-h(t)}^{t-h_{1 m}}\left(h_{1 M}-1 m\right) \dot{z}^{T}(s) U \dot{z}(s) d s \\
& \quad \leq-\beta \int_{t-h(t)}^{t-h_{1 m}}\left(h(t)-h_{1 m}\right) \dot{z}^{T}(s) U \dot{z}(s) d s
\end{aligned}
$$




$$
\begin{aligned}
\leq & -\beta\left[z\left(t-h_{1 m}\right)-z(t-h(t))\right]^{T} U \\
& \times\left[z\left(t-h_{1 m}\right)-z(t-h(t))\right] \\
& -\left(h(t)-h_{1 m}\right) \int_{t-h_{1 M}}^{t-h(t)} \dot{z}^{T}(s) U \dot{z}(s) d s \\
= & -(1-\beta) \int_{t-h_{1 M}}^{t-h(t)}\left(h_{1 M}-h_{1 m}\right) \dot{z}^{T}(s) U \dot{z}(s) d s \\
\leq & -(1-\beta) \int_{t-h_{1 M}}^{t-h(t)}\left(h_{2}-h(t)\right) \dot{z}^{T}(s) U \dot{z}(s) d s \\
\leq & -(1-\beta)\left[z(t-h(t))-z\left(t-h_{1 M}\right)\right]^{T} \\
& \times U\left[z(t-h(t))-z\left(t-h_{1 M}\right)\right] .
\end{aligned}
$$

Therefore from (35)-(36), we obtain

$$
\begin{aligned}
&-\delta \int_{t-h_{1 M}}^{t-h_{1 m}} \dot{z}^{T}(s) U \dot{z}(s) d s \\
& \leq- {\left[z(t-h(t))-z\left(t-h_{1 M}\right)\right]^{T} } \\
& \times U\left[z(t-h(t))-z\left(t-h_{1 M}\right)\right] \\
&- {\left[z\left(t-h_{1 m}\right)-z(t-h(t))\right]^{T} } \\
& \times U\left[z\left(t-h_{1 m}\right)-z(t-h(t))\right] \\
&-\beta\left[z\left(t-h_{1 m}\right)-z(t-h(t))\right]^{T} \\
& \times U\left[z\left(t-h_{1 m}\right)-z(t-h(t))\right] \\
&-(1-\beta)\left[z(t-h(t))-z\left(t-h_{1 M}\right)\right]^{T} \\
& \times U\left[z(t-h(t))-z\left(t-h_{1 M}\right)\right] .
\end{aligned}
$$

By using the following identity relation:

$$
\begin{aligned}
0= & -\dot{e}(t)-A e(t)+C f(e(t))+D g\left(e\left(t-h_{1}(t)\right)\right) \\
& +E \int_{t-k_{1}(t)}^{t} h(e(s)) d s+B_{1} K e(t)+B_{2} K e\left(t-h_{2}(t)\right) \\
& +B_{3} K \int_{t-k_{2}(t)}^{t} e(s) d s \\
= & -P \dot{z}(t)-A P z(t)+C f(e(t))+D g\left(e\left(t-h_{1}(t)\right)\right) \\
& +E \int_{t-k_{1}(t)}^{t} h(e(s)) d s-B_{1} Y z(t)+B_{2}(t) u\left(t-h_{2}(t)\right) \\
& +B_{3} \int_{t-k_{2}(t)}^{t} u(s) d s,
\end{aligned}
$$

we have

$$
\begin{aligned}
0= & -2 \dot{z}^{T}(t) P \dot{z}(t)-2 \dot{z}^{T}(t) A P z(t)+2 \dot{z}^{T}(t) C f(e(t)) \\
& +2 \dot{z}^{T}(t) D g\left(e\left(t-h_{1}(t)\right)\right) \\
& +2 \dot{z}^{T}(t) E \int_{t-k_{1}(t)}^{t} h(e(s)) d s-2 \dot{z}^{T}(t) B_{1} Y z(t) \\
& +2 \dot{z}^{T}(t) B_{2}(t) u\left(t-h_{2}(t)\right)+2 \dot{z}^{T}(t) B_{3} \int_{t-k_{2}(t)}^{t} u(s) d s .
\end{aligned}
$$

By using Propositions 3 and 4, we have

$$
\begin{aligned}
& 2 \dot{z}^{T}(t) C f(e(t)) \\
& \leq \dot{z}^{T}(t) C^{T} U_{1} C \dot{z}(t)+f^{T}(e(t)) U_{1}^{-1} f(e(t)) \\
& \leq \dot{z}^{T}(t) C^{T} U_{1} C \dot{z}(t)+e^{T}(t) F^{T} U_{1}^{-1} F e(t) \\
& =\dot{z}^{T}(t) C^{T} U_{1} C \dot{z}(t)+z^{T}(t) P F^{T} U_{1}^{-1} F P z(t), \\
& 2 \dot{z}^{T}(t) D g\left(e\left(t-h_{1}(t)\right)\right) \\
& \leq \dot{z}^{T}(t) D^{T} U_{2} D \dot{z}(t)+g^{T}\left(e\left(t-h_{1}(t)\right)\right) \\
& \times U_{2}^{-1} g\left(e\left(t-h_{1}(t)\right)\right) \\
& \leq \dot{z}^{T}(t) D^{T} U_{2} D \dot{z}(t)+e^{T}\left(t-h_{1}(t)\right) G^{T} U_{2}^{-1} \\
& \times G e\left(t-h_{1}(t)\right) \\
& =\dot{z}^{T}(t) D^{T} U_{2} D \dot{z}(t)+z^{T}\left(t-h_{1}(t)\right) P G^{T} U_{2}^{-1} \\
& \times G P z\left(t-h_{1}(t)\right), \\
& 2 \dot{z}^{T}(t) E \int_{t-k_{1}(t)}^{t} h(e(s)) d s \\
& \leq 2 k_{1} e^{2 \alpha k_{1}} \dot{z}^{T}(t) E^{T} U_{3} E \dot{z}(t) \\
& +\frac{1}{2 k_{1}} e^{-2 \alpha k_{1}}\left(\int_{t-k_{1}(t)}^{t} h(e(s)) d s\right)^{T} \\
& \times U_{3}^{-1}\left(\int_{t-k_{1}(t)}^{t} h(e(s)) d s\right) \\
& \leq 2 k_{1} e^{2 \alpha k_{1}} \dot{z}^{T}(t) E^{T} U_{3} E \dot{z}(t) \\
& +\frac{e^{-2 \alpha k_{1}}}{2} \int_{t-k_{1}(t)}^{t} h^{T}(e(s)) U_{3}^{-1} h(e(s)) d s, \\
& 2 \dot{z}^{T}(t) B_{2}(t) u\left(t-h_{2}(t)\right) \\
& \leq 3 e^{2 \alpha h_{2}} \dot{z}^{T}(t) B_{2}^{T} S_{1} B_{2} \dot{z}(t) \\
& +\frac{e^{-2 \alpha h_{2}}}{3} u^{T}\left(t-h_{2}(t)\right) S_{1}^{-1} u\left(t-h_{2}(t)\right), \\
& 2 \dot{z}^{T}(t) B_{3} \int_{t-k_{2}(t)}^{t} u(s) d s
\end{aligned}
$$




$$
\begin{aligned}
\leq & 2 k_{2} e^{2 \alpha k_{2}} \dot{z}^{T}(t) B_{3}^{T} S_{2} B_{3} \dot{z}(t) \\
& +\frac{e^{-2 \alpha k_{2}}}{2 k_{2}}\left(\int_{t-k_{2}(t)}^{t} u(s) d s\right)^{T} S_{2}^{-1}\left(\int_{t-k_{1}(t)}^{t} u(s) d s\right) \\
\leq & 2 k_{2} e^{2 \alpha k_{2}} \dot{z}^{T}(t) B_{3}^{T} S_{2} B_{3} \dot{z}(t) \\
& +\frac{e^{-2 \alpha k_{2}}}{2} \int_{t-k_{2}(t)}^{t} u^{T}(s) S_{2}^{-1} u(s) d s .
\end{aligned}
$$

From (29)-(40), we obtain

$$
\begin{aligned}
\dot{V}(t, e(t))+2 \alpha V(t, e(t)) \\
\leq \xi^{T}(t)\left((1-\beta) \mathscr{M}_{1}+\beta \mathscr{M}_{2}\right) \xi(t)+z^{T}(t) \mathscr{M}_{3} z(t) \\
\quad+\dot{z}^{T}(t) \mathscr{M}_{4} \dot{z}(t)+z^{T}\left(t-h_{1}(t)\right) \mathscr{M}_{5} z\left(t-h_{1}(t)\right) \\
\quad-e^{T}(t) \mathscr{M}_{6} e(t),
\end{aligned}
$$

where

$$
\begin{aligned}
\mathscr{M}_{3}= & -0.1 R\left(e^{-2 \alpha h_{1 m}}+e^{-2 \alpha h_{1 M}}\right)+2 P F^{T} U_{1}^{-1} F P \\
& +P H^{T} U_{3}^{-1} H P+2 e^{-2 \alpha h_{2}} Y^{T} S_{1}^{-1} Y \\
& +P Q_{1} P+Y^{T} Q_{2} Y, \\
\mathscr{M}_{4}= & 0.1 P+h_{2}^{2} Y^{T} S_{1}^{-1} Y, \\
\mathscr{M}_{5}= & -0.1 e^{-2 \alpha h_{1 M}} U+2 P G^{T} U_{2}^{-1} G P, \\
\mathscr{M}_{6}= & Q_{1}+P^{-1} Y^{T} Q_{2} Y P^{-1}, \\
\xi(t)= & {\left[z(t), \dot{z}(t), z\left(t-h_{1 m}\right), z(t-h(t)), z\left(t-h_{1 M}\right)\right] . }
\end{aligned}
$$

Since $0 \leq \beta \leq 1,(1-\beta) \mathscr{M}_{1}+\beta \mathscr{M}_{2}$ is a convex combination of $\mathscr{M}_{1}$ and $\mathscr{M}_{2}$. Therefore, $(1-\beta) \mathscr{M}_{1}+\beta \mathscr{M}_{2}<0$ is equivalent to $\Gamma_{1}<0$ and $\Gamma_{2}<0$. Applying Schur complement lemma, Proposition 5, the inequalities $\mathscr{M}_{3}<0, \mathscr{M}_{4}<0$, and $\mathscr{M}_{5}<0$ are equivalent to $\Gamma_{3}<0, \Gamma_{4}<0$, and $\Gamma_{5}<0$, respectively. Thus, from (18)-(21) and (41), we obtain

$$
\dot{V}(t, e(t))+2 \alpha V(t, e(t)) \leq-e^{T}(t) \mathscr{M}_{6} e(t), \quad \forall t \geq 0 .
$$

Since $\mathscr{M}_{6}>0$, we have

$$
\dot{V}(t, e(t)) \leq-2 \alpha V(t, e(t)), \quad \forall t \geq 0 .
$$

Integrating both sides of (44) from 0 to $t$, we obtain

$$
V(t, e(t)) \leq V(\phi(t)) e^{-2 \alpha t}, \quad \forall t \geq 0 .
$$

Furthermore, taking condition (28) into account, we have

$$
\lambda_{1}\|e(t, \phi)\|^{2} \leq V(t, e(t)) \leq V(\phi(t)) e^{-2 \alpha t} \leq \lambda_{2} e^{-2 \alpha t}\|\phi\|^{2} .
$$

Because $V(t, e(t))$ is radially unbounded, by the LyapunovKrasovskii theorem and the solution $\|e(t, \phi)\|$ of the error system (6) satisfy

$$
\|e(t, \phi)\| \leq \sqrt{\frac{\lambda_{2}}{\lambda_{1}}} e^{-\alpha t}\|\phi\|, \quad \forall t \geq 0,
$$

which implies the exponential stability of the error system (6) under the controller (H1). Consequentially, the controlled slave system (1) is synchronized with the master system (2).

Furthermore, from $(43)$ and $V(t, e(t))>0$, we have

$$
\dot{V}(t, e(t)) \leq-e^{T}(t) \mathscr{M}_{5} e(t), \quad \forall t \geq 0 .
$$

Integrating both sides of (48) from 0 to $t$, we obtain

$$
\int_{0}^{t} e^{T}(t) \mathscr{M}_{5} e(t) d t \leq V(0, \phi)-V(t, e(t)) \leq V(0, \phi),
$$

$$
\forall t \geq 0
$$

due to $V(t, e(t)) \geq 0$. Hence

$$
\int_{0}^{t} e^{T}(t) \mathscr{M}_{5} e(t) d t \leq V(0, \phi)=\lambda_{2}\|\phi\|^{2} .
$$

Given $t \rightarrow \infty$, we obtain

$$
J=\int_{0}^{\infty} e^{T}(t) \mathscr{M}_{5} e(t) d t \leq \lambda_{2}\|\phi\|^{2}=J^{*} .
$$

The proof is completed.

Remark 7. In our main results, guaranteed cost synchronization problem for cellular neural networks with interval nondifferentiable time-varying and distributed timevarying delays is considered. We first construct the improved Lyapunov-Krasovskii functionals $V(t, e(t))$ as shown in (26). We give sufficient conditions for the exponential synchronization of the error systems are independent on the derivatives of the time-varying delays and without introducing any free-weighting matrices turn out to be less conservative with fewer matrix variables than see $[7,9,20,35,38,39,41]$.

Remark 8 . In most results on guaranteed cost synchronization problem for cellular neural networks, authors have considered only activation functions with time-varying delay [21, 22]. But in our works, we have considered a more complicated problem, namely, guaranteed cost synchronization of cellular neural networks with various activation functions and mixed time-varying delays in state and feedback control term simultaneously. To the best of our knowledge, our results are among the first results on guaranteed cost synchronization of cellular neural networks with various activation functions and mixed time-varying delays using hybrid feedback control. Therefore, our stability conditions are less conservative than other existing results $[21,22]$.

\section{Numerical Examples}

In this section, we now provide an example to show the effectiveness of the result in Theorem 6 . 
Example 9. Consider the cellular neural networks with various activation functions and mixed time-varying delays using hybrid feedback control with the following parameters:

$$
\begin{aligned}
\dot{x}(t)= & -A x(t)+C \tilde{f}(x(t))+D \tilde{g}\left(x\left(t-h_{1}(t)\right)\right) \\
+ & E \int_{t-k_{1}(t)}^{t} \tilde{h}(x(s)) d s+I(t), \\
& x(t)=\phi_{1}(t), \quad t \in[-d, 0], \\
\dot{y}(t)=- & A y(t)+C \tilde{f}(y(t))+D \tilde{g}\left(y\left(t-h_{1}(t)\right)\right) \\
+ & E \int_{t-k_{1}(t)}^{t} \tilde{h}(y(s)) d s+I(t)+B_{1} u(t) \\
+ & B_{2} u\left(t-h_{2}(t)\right)+B_{3} \int_{t-k_{2}(t)}^{t} u(s) d s, \\
& y(t)=\phi_{2}(t), \quad t \in[-d, 0],
\end{aligned}
$$

where

$$
\begin{gathered}
A=\left[\begin{array}{ll}
1 & 0 \\
0 & 1
\end{array}\right], \quad C=\left[\begin{array}{ll}
0.2 & -0.2 \\
0.1 & -0.3
\end{array}\right], \quad D=\left[\begin{array}{cc}
0.2 & 0.1 \\
-0.1 & 0.2
\end{array}\right], \\
E=\left[\begin{array}{cc}
0.1 & 0.2 \\
-0.5 & 0.1
\end{array}\right], \quad F=\left[\begin{array}{cc}
0.5 & 0 \\
0 & 0.3
\end{array}\right], \quad G=\left[\begin{array}{cc}
0.5 & 0 \\
0 & 0.4
\end{array}\right], \\
H=\left[\begin{array}{cc}
0.4 & 0 \\
0 & 0.2
\end{array}\right], \quad B_{1}=\left[\begin{array}{ll}
4 & 0 \\
0 & 4
\end{array}\right], \quad B_{2}=\left[\begin{array}{ll}
1 & 0 \\
0 & 1
\end{array}\right], \\
B_{3}=\left[\begin{array}{ll}
1 & 0 \\
0 & 1
\end{array}\right], \\
\phi_{1}(t)=[-0.4 \cos t, 0.5 \cos t], \quad \phi_{2}(t)=[\sin t, \sin t] .
\end{gathered}
$$

Solution 1. From the conditions (18)-(22) of Theorem 6, we let $\alpha=0.02, h_{1 m}=0.1, h_{1 M}=0.3, h_{2}=0.3, k_{1}=0.3$, $k_{2}=0.2, Q_{1}=\left[\begin{array}{ll}3 & 0 \\ 0 & 2\end{array}\right]$, and $Q_{2}=\left[\begin{array}{ll}2 & 0 \\ 0 & 1\end{array}\right]$. By using the LMI Toolbox in MATLAB, we obtain

$$
\begin{aligned}
& P=\left[\begin{array}{cc}
00.0789 & 0.0066 \\
0.0066 & 0.1024
\end{array}\right], \quad Q=\left[\begin{array}{ll}
0.0108 & 0.0039 \\
0.0039 & 0.0196
\end{array}\right], \\
& R=\left[\begin{array}{ll}
0.2139 & 0.0388 \\
0.0388 & 0.2779
\end{array}\right], \quad U=\left[\begin{array}{ll}
0.1375 & 0.0336 \\
0.0336 & 0.2546
\end{array}\right], \\
& S_{1}=\left[\begin{array}{ll}
0.0009 & 0.0002 \\
0.0002 & 0.0012
\end{array}\right], \quad S_{2}=\left[\begin{array}{ll}
0.0012 & 0.0009 \\
0.0009 & 0.0027
\end{array}\right], \\
& U_{1}=\left[\begin{array}{cc}
0.1649 & 0 \\
0 & 0.0935
\end{array}\right], \quad U_{2}=\left[\begin{array}{cc}
0.2482 & 0 \\
0 & 0.1464
\end{array}\right] \text {, } \\
& U_{3}=\left[\begin{array}{cc}
0.2491 & 0 \\
0 & 0.0336
\end{array}\right], \quad Y=10^{-3}\left[\begin{array}{ll}
0.1491 & 0.1249 \\
0.0726 & 0.3067
\end{array}\right] \text {, } \\
& K=\left[\begin{array}{ll}
-0.0018 & -0.0011 \\
-0.0007 & -0.0030
\end{array}\right] \text {, }
\end{aligned}
$$

and accordingly the feedback control is $u(t)=$ $\left[\begin{array}{ll}-0.0018 & -0.0011 \\ -0.0007 & -0.0030\end{array}\right](y(t)-x(t))$. Thus, is 0.02 exponentially synchronization and the value $\sqrt{\lambda_{2} / \lambda_{1}}=5.2860$, so the solution of the closed-loop system satisfies

$$
\left\|y\left(t, \phi_{2}\right)-x\left(t, \phi_{1}\right)\right\| \leq 5.2860 e^{-0.02 t}\|\phi\|, \quad \forall t \in \mathbb{R}^{+},
$$

and the optimal guaranteed cost of the closed-loop system is as follows:

$$
J \leq J^{*}=4.6365 \text {. }
$$

We let $h_{1}(t)=0.1+0.2|\sin t|, h_{2}(t)=0.3 e^{|\sin t|}, k_{1}(t)=$ $0.3|\cos t|, k_{2}(t)=0.2 e^{|\cos t|}, \phi_{1}(t)=[-0.4 \cos t, 0.5 \cos t]$, $\phi_{2}(t)=[\sin t, \sin t]$, for all $t \in[-0.3,0]$, and the activation functions as follows:

$$
\begin{gathered}
f_{1}\left(x_{1}(t)\right)=0.25\left(\left|x_{1}(t)+1\right|-\left|x_{1}(t)-1\right|\right), \\
f_{2}\left(x_{2}(t)\right)=0.15\left(\left|x_{1}(t)+1\right|-\left|x_{1}(t)-1\right|\right), \\
g_{1}\left(x_{1}(t)\right)=0.25\left(\left|x_{1}(t)+1\right|-\left|x_{1}(t)-1\right|\right), \\
g_{2}\left(x_{2}(t)\right)=0.2\left(\left|x_{2}(t)+1\right|-\left|x_{2}(t)-1\right|\right), \\
h_{1}\left(x_{1}(s)\right)=0.4 \tanh \left(-6 x_{1}(s)\right), \\
h_{2}\left(x_{2}(s)\right)=0.2 \tanh \left(7 x_{2}(s)\right) .
\end{gathered}
$$

Figure 1 shows the trajectories of solutions $e_{1}(t)$ and $e_{2}(t)$ of the cellular neural networks with various activation functions and mixed time-varying delays without hybrid feedback control $(u(t)=0)$. Figure 2 shows the trajectories of solutions $e_{1}(t)$ and $e_{2}(t)$ of the cellular neural networks with various activation functions and mixed time-varying delays with hybrid feedback control $u(t)=\left[\begin{array}{lll}-0.0018 & -0.0011 \\ -0.0007 & -0.0030\end{array}\right](y(t)-x(t))$.

Remark 10. The advantage of Example 9 is the lower bound of the delay $h_{m} \neq 0$ and interval time-varying delay and distributed time-varying delay are nondifferentiable. Moreover, in these examples we still investigate various activation functions and mixed time-varying delays in state and feedback control term simultaneously; hence the synchronization conditions derived in $[21,22]$ cannot be applied to these examples.

\section{Conclusions}

In this paper, we have investigated the exponential synchronization of cellular neural networks with various activation functions and mixed time-varying delays via hybrid feedback control. The interval time-varying delay function is not necessary to be differentiable which allows time-delay function to be a fast time-varying function. A new class of LyapunovKrasovskii functional is constructed in new delay-dependent sufficient conditions for the exponential synchronization of the error systems, which have been derived by a set of LMIs without introducing any free-weighting matrices. The optimal guaranteed cost control with linear error hybrid feedback is turned into the solvable problem of a set of LMIs. Simulation results have been given to illustrate the effectiveness of the proposed method. 


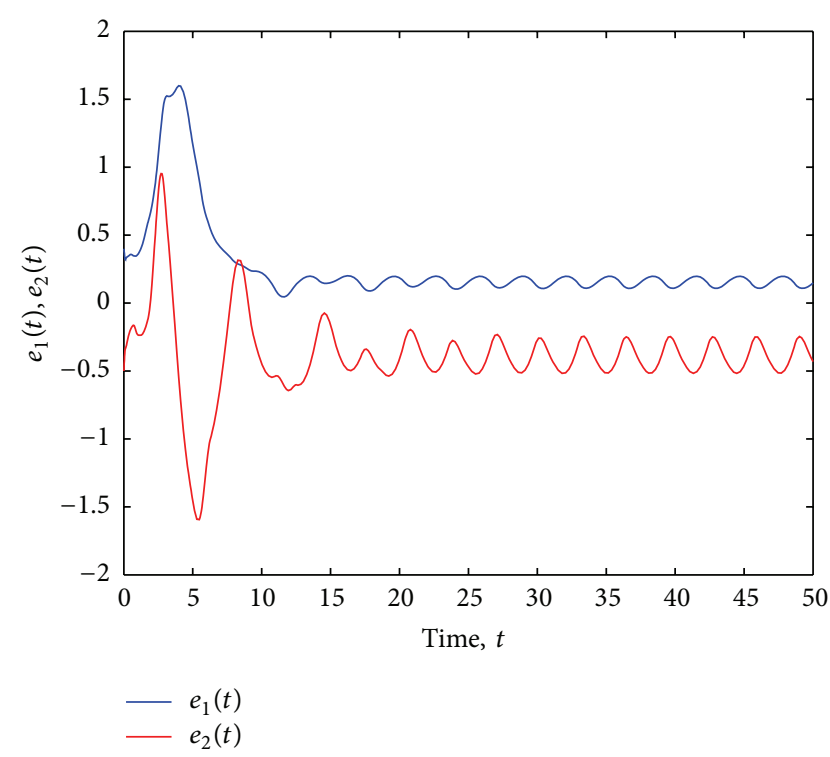

FIGURE 1: Synchronization error curves of the master system (52) and the slave system (53) without hybrid feedback control.

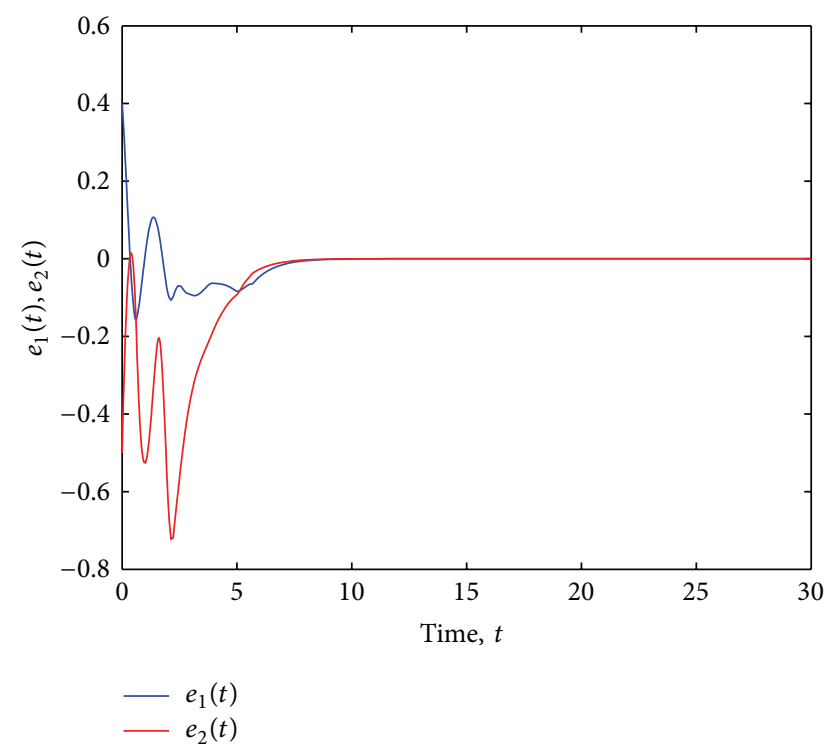

FIGURE 2: Synchronization error curves of the master system (52) and the slave system (53) with hybrid feedback control input.

\section{Acknowledgments}

The authors thank anonymous reviewers for their valuable comments and suggestions. This work is supported by Coordinating Center for Thai Government Science and Technology Scholarship Students (CSTS) of the National Science and Technology Development Agency (NSTDA) (SCH-NR2011841), the Center of Excellence in Mathematics, Thailand, and Commission for Higher Education, Thailand, and the Thailand Research Fund through the Royal Golden Jubilee Ph.D. Program (Grant no. PHD/0355/2552) to W. Weera and P. Niamsup is acknowledged.

\section{References}

[1] M. M. Gupta, L. Jin, and N. Homma, Static and Dynamic Neural Networks: From Fundamentalsto Advanced Theory, Wiley, New York, NY, USA, 2003.

[2] L. M. Pecora and T. L. Carroll, "Synchronization in chaotic systems," Physical Review Letters, vol. 64, no. 8, pp. 821-824, 1990.

[3] J. Liang and J. Cao, "Global asymptotic stability of bi-directional associative memory networks with distributed delays," Applied Mathematics and Computation, vol. 152, no. 2, pp. 415-424, 2004.

[4] H. Zhao, "Global asymptotic stability of Hopfield neural network involving distributed delays," Neural Networks, vol. 17, no. 1, pp. 47-53, 2004.

[5] P. Balasubramaniam and V. Vembarasan, "Synchronization of recurrent neural networks with mixed time-delays via output coupling with delayed feedback," Nonlinear Dynamics, vol. 70, no. 1, pp. 677-691, 2012.

[6] P. Balasubramaniam, R. Chandran, and S. Jeeva Sathya Theesar, "Synchronization of chaotic nonlinear continuous neural networks with time-varying delay," Cognitive Neurodynamics, vol. 5, pp. 361-371, 2011.

[7] M. V. Thuan, "Guaranteed cost control of neural networks with various activation functions and mixed time-varying delays in state and control," Differential Equations and Control Processes, vol. 3, pp. 18-29, 2011.

[8] W. Weera and P. Niamsup, "Exponential stabilization of neutraltype neural networks with interval nondifferentiable and distributed time-varying delays," Abstract and Applied Analysis, vol. 2012, Article ID 101426, 21 pages, 2012.

[9] V. N. Phat and H. Trinh, "Exponential stabilization of neural networks with varous activation functions and mixed timevarying delays," IEEE Transactions on Neural Networks, vol. 21, pp. 1180-1184, 2010.

[10] J. Cai, X. Wu, and S. Chen, "Synchronization criteria for nonautonomous chaotic systems with sinusoidal state error feedback control," Physica Scripta, vol. 75, pp. 379-387, 2007.

[11] T. Botmart and P. Niamsup, "Adaptive control and synchronization of the perturbed Chua's system," Mathematics and Computers in Simulation, vol. 75, no. 1-2, pp. 37-55, 2007.

[12] S. Jeeva Sathya Theesar, S. Banerjee, and P. Balasubramaniam, "Adaptive synchronization in noise perturbed chaotic systems," Physica Scripta, vol. 85, no. 6, Article ID 065010, 2012.

[13] J. Cao, D. W. C. Ho, and Y. Yang, "Projective synchronization of a class of delayed chaotic systems via impulsive control," Physics Letters A, vol. 373, no. 35, pp. 3128-3133, 2009.

[14] J. Lu, D. W. C. Ho, J. Cao, and J. Kurths, "Exponential synchronization of linearly coupled neural networks with impulsive disturbances," IEEE Transactions on Neural Networks, vol. 22, pp. 329-335, 2011.

[15] G. H. Li, S. P. Zhou, and K. Yang, "Generalized projective synchronization between two different chaotic systems using active backstepping control," PPhysics Letters A, vol. 355, pp. 326-330, 2006.

[16] H. Guo and S. Zhong, "Synchronization criteria of time-delay feedback control system with sector-bounded nonlinearity," Applied Mathematics and Computation, vol. 191, no. 2, pp. 550559, 2007.

[17] T. Botmart, P. Niamsup, and X. Liu, "Synchronization of nonautonomous chaotic systems with time-varying delay via delayed feedback control," Communications in Nonlinear Science and Numerical Simulation, vol. 17, no. 4, pp. 1894-1907, 2012. 
[18] S. Jeeva Sathya Theesar, S. Banerjee, and P. Balasubramaniam, "Synchronization of chaotic systems under sampled-data control," Nonlinear Dynamics, vol. 70, pp. 1977-1987, 2012.

[19] S. S. L. Chang and T. K. C. Peng, "Adaptive guaranteed cost control of systems with uncertain parameters," IEEE Transactions on Automatic Control, vol. 17, no. 4, pp. 474-483, 1972.

[20] M. V. Thuan and V. N. Phat, "Optimal guaranteed cost control of linear systems with mixed interval time-varying delayed state and control," Journal of Optimization Theory and Applications, vol. 152, no. 2, pp. 394-412, 2012.

[21] J. Tu, H. He, and P. Xiong, "Guaranteed cost synchronous control of time-varying delay cellular neural networks," Neural Computing and Applications, vol. 22, no. 1, pp. 103-110, 2013.

[22] J. Tu and H. He, "Guaranteed cost synchronization of chaotic cellular neural networks with time-varying delay," Neural Computation, vol. 24, no. 1, pp. 217-233, 2012.

[23] B. Chen, X. Liu, and S. Tong, "Guaranteed cost control of timedelay chaotic systems via memoryless state feedback," Chaos, Solitons \& Fractals, vol. 34, no. 5, pp. 1683-1688, 2007.

[24] T. H. Lee, J. H. Park, D. H. Ji, O. M. Kwon, and S. M. Lee, “Guaranteed cost synchronization of a complex dynamical network via dynamic feedback control," Applied Mathematics and Computation, vol. 218, no. 11, pp. 6469-6481, 2012.

[25] C. J. Cheng, T. L. Liao, J. J. Yan, and C. C. Hwang, "Exponential synchronization of a class of neural networks with time-varying delays," IEEE Transactions on Systems, Man, and Cybernetics B, vol. 36, pp. 209-215, 2006.

[26] X. Gao, S. Zhong, and F. Gao, "Exponential synchronization of neural networks with time-varying delays," Nonlinear Analysis. Theory, Methods \& Applications, vol. 71, no. 5-6, pp. 2003-2011, 2009.

[27] S. Jeeva Sathya Theesar, R. Chandran, and P. Balasubramaniam, "Delay-dependent exponential synchronization criteria for chaotic neural networks with time-varying delays," Brazilian Journal of Physics, vol. 42, pp. 207-218, 2012.

[28] Y. Sun, J. Cao, and Z. Wang, "Exponential synchronization of stochastic perturbed chaotic delayed neural networks," Neurocomputing, vol. 70, no. 13-15, pp. 2477-2485, 2007.

[29] T. Li, S.-M. Fei, Q. Zhu, and S. Cong, "Exponential synchronization of chaotic neural networks with mixed delays," Neurocomputing, vol. 71, no. 13-15, pp. 3005-3019, 2008.

[30] T. Li, S.-M. Fei, and K.-J. Zhang, "Synchronization control of recurrent neural networks with distributed delays," Physica A, vol. 387, no. 4, pp. 982-996, 2008.

[31] H. R. Karimi and H. Gao, "New delay-dependent exponential $H_{\infty}$ synchronization for uncertain neural networks with mixed time delays," IEEE Transactions on Systems, Man, and Cybernetics B, vol. 40, pp. 173-185, 2010.

[32] Z.-G. Wu, P. Shi, H. Su, and J. Chu, "Exponential synchronization of neural networks with discrete and distributed delays under time-varying sampling," IEEE Transactions on Neural Networks, vol. 23, pp. 1368-1376, 2012.

[33] Q. Song, "Design of controller on synchronization of chaotic neural networks with mixed time-varying delays," Neurocomputing, vol. 72, no. 13-15, pp. 3288-3295, 2009.

[34] T. Botmart, P. Niamsup, and V. N. Phat, "Delay-dependent exponential stabilization for uncertain linear systems with interval non-differentiable time-varying delays," Applied Mathematics and Computation, vol. 217, no. 21, pp. 8236-8247, 2011.

[35] O. M. Kwon and J. H. Park, "Exponential stability analysis for uncertain neural networks with interval time-varying delays,"
Applied Mathematics and Computation, vol. 212, no. 2, pp. 530541, 2009.

[36] J. Tian and X. Zhou, "Improved asymptotic stability criteria for neural for networks with interval time-varying delay," Expert Systems with Applications, vol. 37, pp. 7521-7525, 2010.

[37] D. Wang and W. Wang, "Delay-dependent robust exponential stabilization for uncertain systems with interval time-varying delays," Journal of Control Theory and Applications, vol. 7, no. 3, pp. 257-263, 2009.

[38] J. H. Park, "Further result on asymptotic stability criterion of cellular neural networks with time-varying discrete and distributed delays," Applied Mathematics and Computation, vol. 182, no. 2, pp. 1661-1666, 2006.

[39] J. H. Park, "On global stability criterion of neural networks with continuously distributed delays," Chaos, Solitons and Fractals, vol. 37, no. 2, pp. 444-449, 2008.

[40] J. H. Park, "An analysis of global robust stability of uncertain cellular neural networks with discrete and distributed delays," Chaos, Solitons and Fractals, vol. 73, pp. 2789-2792, 2010.

[41] J. H. Park and H. J. Cho, "A delay-dependent asymptotic stability criterion of cellular neural networks with time-varying discrete and distributed delays," Chaos, Solitons and Fractals, vol. 33, no. 2, pp. 436-442, 2007.

[42] K. Gu, V. L. Kharitonov, and J. Chen, Stability of Time-Delay System, Birkhäauser, Boston, Mass, USA, 2003. 


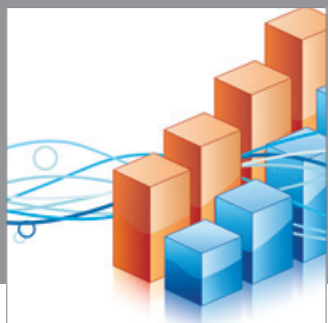

Advances in

Operations Research

mansans

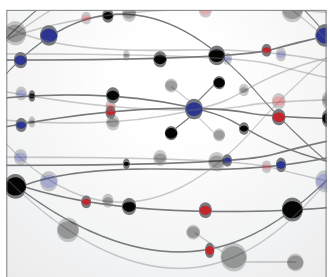

The Scientific World Journal
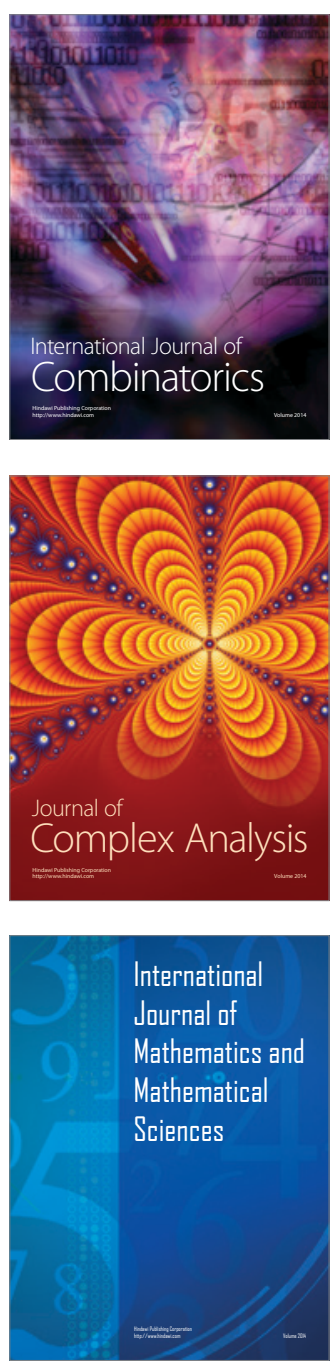
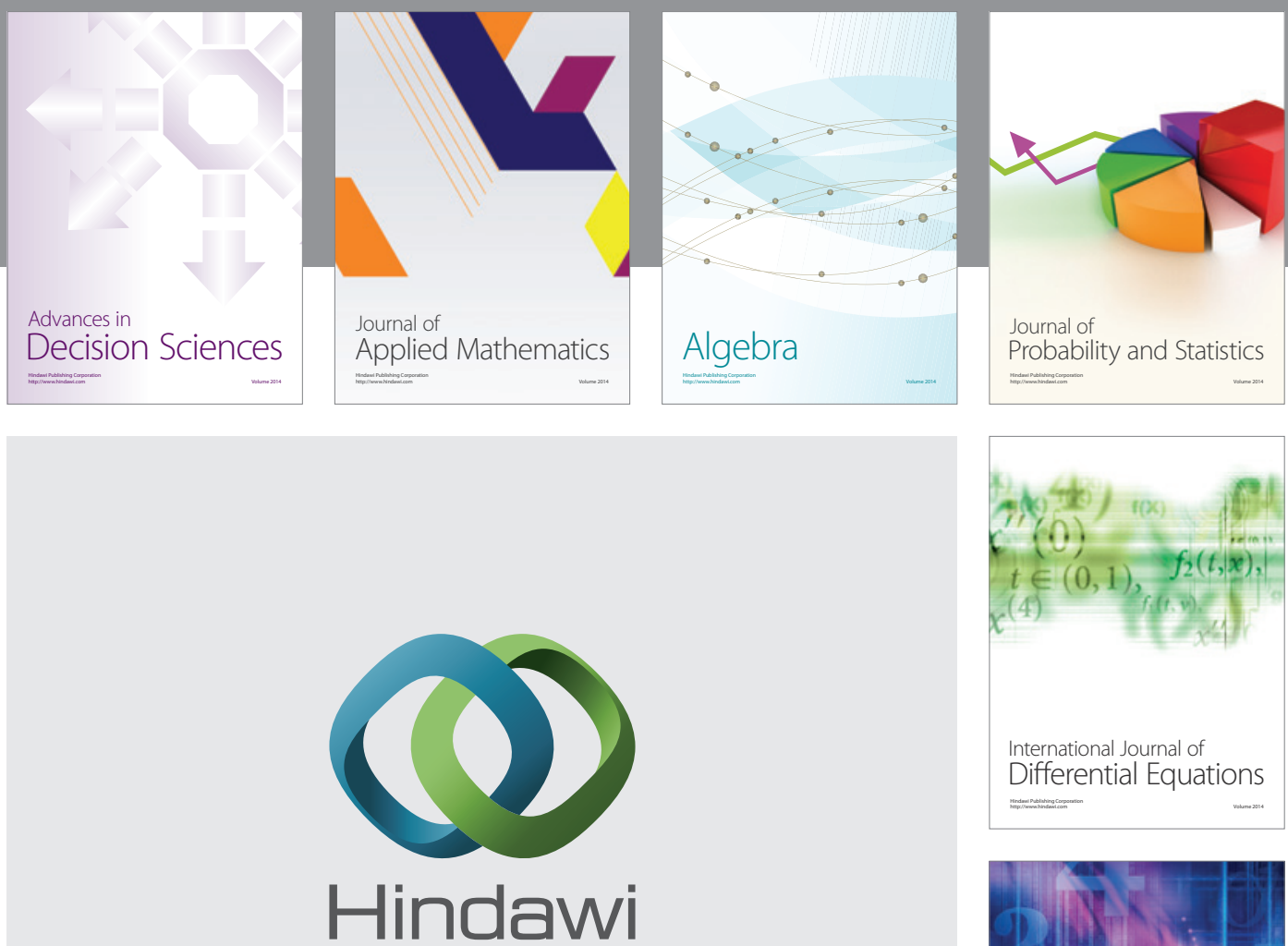

Submit your manuscripts at http://www.hindawi.com
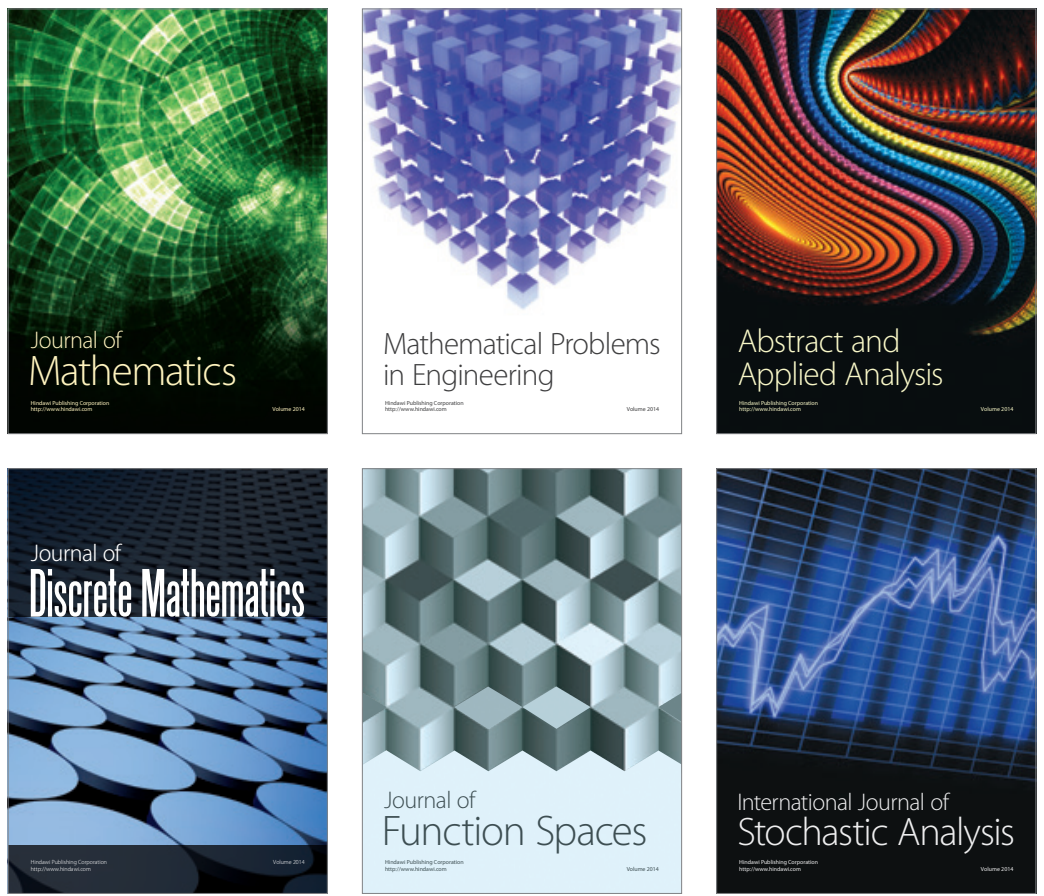

Journal of

Function Spaces

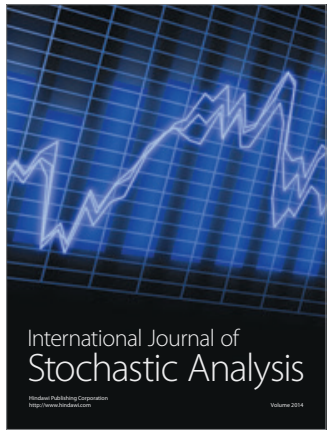

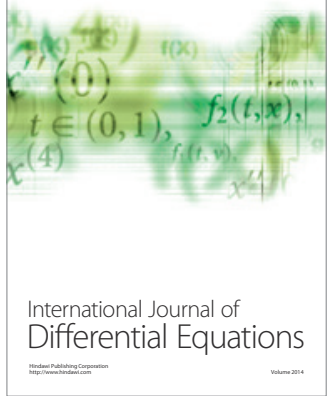
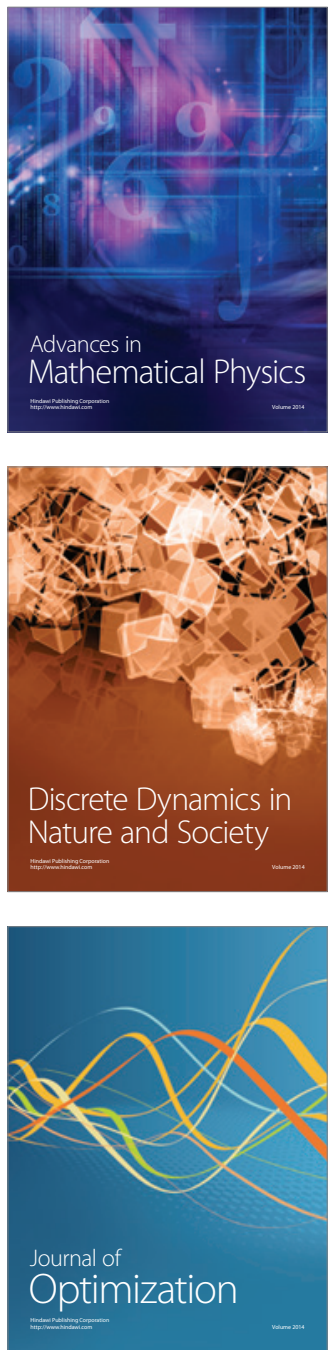